Résumés des conférences et travaux

\title{
Sources et histoire de la tradition Sanskrite
}

Jan E. M. Houben

\section{OpenEdition \\ Journals}

Édition électronique

URL : https://journals.openedition.org/ashp/908

DOI : 10.4000/ashp.908

ISSN : 1969-6310

Éditeur

Publications de l'École Pratique des Hautes Études

\section{Édition imprimée}

Date de publication : 1 octobre 2009

Pagination : 352-356

ISSN : 0766-0677

\section{Référence électronique}

Jan E. M. Houben, «Sources et histoire de la tradition Sanskrite ", Annuaire de l'École pratique des hautes études (EPHE), Section des sciences historiques et philologiques [En ligne], 140 | 2009, mis en ligne le 03 novembre 2009, consulté le 06 juillet 2021. URL : http://journals.openedition.org/ashp/908 ; DOl : https://doi.org/10.4000/ashp.908 


\title{
SOURCES ET HISTOIRE DE LA TRADITION SANSKRITE
}

\author{
Directeur d'études : M. Jan E. M. Houben
}

Programme de l'année 2007-2008 : I. Le Prakriyāsarvasva : une grammaire pāninéenne du sanskrit « vivant ». - II. Narratif et rituel dans la prose védique : la Maitrāyan̄isaminhitā. — III. Ayurveda : textes pré-modernes.

\section{Le Prakriyāsarvasva : une grammaire pāninéenne du sanskrit « vivant »}

Aux environs de 350 av. notre ère, Pāṇini composa une grammaire remarquablement compacte et complète de la langue des Vedas et de la langue parlée de haut statut (que nous appellons maintenant le sanskrit). Les autres grammaires de l'époque, probablement pas aussi complètes que celle de Pạninini, furent poussées dans l'oubli. Les premiers commentaires critiques sur la grammaire de Pānini, dans sa forme pleine d'environ 4000 règles et de quelques annexes telles que la liste d'environ 2000 racines, sont ceux de Kātyāyana et Patañjali (env. $\mathrm{III}^{\mathrm{e}}$ et $\mathrm{II}^{\mathrm{e}}$ siècle av. notre ère). Les siècles suivants ont connu plusieurs ajouts et adaptations des règles et des listes de la grammaire de Pāninini. Plusieurs formes et interprétations de la grammaire sanskrite se sont développées. De nouvelles grammaires ont été composées avec de nouveaux titres, bien qu'elles suivent, dans une très grande mesure, la grammaire de Pāṇini. Aussi la grammaire pāṇinéenne n'est pas seulement une discipline difficile et technique; elle a été en outre, à travers les millénaires, un champ vibrant de discussion érudite et de production textuelle scientifique. Grâce aux changements proposés et acceptés par quelques uns ou par tous les transmetteurs de la grammaire, nous en avons plusieurs versions dont la forme actuellement la plus acceptée est celle de la Siddhāntakaumudī de Bhatṭoji Dīkșita. L'objet de la grammaire pāṇinéenne de Bhaț̣oji (du XIV ${ }^{\mathrm{e}}-\mathrm{XVII}^{\mathrm{e}}$ siècle), environ deux millénaires après Pāṇini, est la langue sanskrite telle qu'elle était connue de Pāṇini, Kātyāyana et Patañjali - à savoir le sanskrit jusqu'à env. le II $^{\mathrm{e}}$ siècle av. notre ère. Des formes linguistiques plus tardives qui ne sont pas autorisées par les règles ou reconnues par ces trois grammairiens sont en principe rejetées. Une conséquence secondaire de cette démarche est que des textes sanskrits importants dans l'histoire culturelle de l'Inde, tel que le Mahābhārata, deviennent « non-grammaticaux ». Ceci peut sembler un désavantage, mais il constitue plutôt un avantage pour les brahmanes de conviction śivaite dans leurs disputes avec les brahmanes de conviction viṣnuite (ces derniers comprenant plusieurs opposants de Bhattojii, y compris ... son maître direct Śeșa-krș̣na), parce que c'est surtout pour les viṣṇuites que le Mahābhārata constitue une source d'autorité pour les rites et la religion. Dans cette controverse sousjacente à la science linguistique de la grammaire, Bhattoji et d'autres grammairiens de sa famille, et plus tard de son école, manifestent leur préférence, par exemple, à travers le nom qu'ils donnent aux pratyāhāra-sūtra, le répertoire des phonèmes acceptés par la grammaire : ils les appellent māheśvara-sūtra pour évoquer la légende, actuel- 
lement assez connue, selon laquelle ce répertoire n'a pas d'auteur humain, mais descend directement du dieu Maheśvara, c'est à dire de Śiva. Une légende alternative était proposée un millénaire avant Bhatṭoji par les bouddhistes, selon qui les pratyāhārasūtra dérivent plutôt du Bodhisatva Avalokiteśvara. Les viṣnuites, par contre, continuaient à renvoyer à ce répertoire de phonèmes exclusivement par leur nom technique pratyāhāra-sūtra «formule pour la "condensation" des phonèmes ».

Au cours du séminaire, nous avons poursuivi l'étude des « méta-règles » de la « section des principes » (nyāya-khaṇdah) du Prakriyā-sarvasva de Nārāyaṇa Bhațta, contemporain plus âgé de Bhaț̣oji Dīkṣita. Contrairement à la Siddhānta-kaumudī de Bhatṭoji, le Prakriyā-sarvasva a été largement négligé et même oublié aussi bien en Inde que par les indianistes internationaux. Contrairement à la Siddhānta-kaumudī, en outre, le Prakriyā-sarvasva adopte une attitude libérale et accepte des développements linguistiques postérieurs à l'époque de Kātyāyana et de Patañjali. L'ironie, c'est que Bhattoji s'efforcant au maximum de conserver la grammaire de Pāninini et de refuter les grammairiens qui acceptent des formes linguistiques relativement tardives finit par rater, dans cet effort même, un aspect crucial de l'esprit pāṇinéen : un libéralisme linguistique qui se manifeste par des renvois aux autres grammairiens et aux règles optionnelles. Par contre, c'est la grammaire pāṇinéenne de Nārāyaṇa Bhaț̣a défendant des grammairiens et des formes linguistiques plus tardives (post-Patañjali) qui est plus proche de Pāninini du point de vue de ce libéralisme linguistique. Le premier principe de la « section des principes » du Prakriyā-sarvasva est significatif dans ce contexte : vivakṣātah kārakāṇi, qu'on peut traduire comme suit : « Les kāraka (rôles dans une action tel qu'agent, instrument, etc.) sont (attribués aux objets figurant dans la situation exprimée dans la phrase) selon le désir (du locuteur) d'exprimer. » Le fait que Nārāyaṇa met les kārakas et le désir d'exprimer au début est unique. Ni Nāgeśa ni Vyāḍi mentionnent parmi leurs paribhāṣā ce principe qu'on trouve pourtant dans quelques autres grammaires (par ex., dans le Sarasvatī-kaṇṭābharaṇa de Bhoja et, avant lui, sous une forme un peu différent - vivakșātah kārakāvasāyaḥ - dans l'œuvre du grammairien bouddhiste Candragomin) mais jamais au début. L'importance attribuée par Nārāyaṇa à la vivakṣā montre sa confiance au locuteur, l'importance attribuée aux kārakas montre que la phrase - plutôt que le mot - est le point de départ. L'ensemble confirme que pour Nārāyaṇa Bhațta le sanskrit était une langue érudite, bien sûr, mais aussi « vivante » : une langue dont le développement ne s'arrête pas avec Pāninini, Kātyāyana et Patañjali, une langue pour laquelle on peut toujours se fier au désir d'exprimer - à l'intuition linguistique - des locuteurs. Parmi les autres principes qui ont retenu notre attention se trouve le principe $\mathrm{n}^{\circ} 20$, asiddham bahir-angam antar-ange « Ce qui est "externe" n'est pas effectué pour ce qui est "interne" », ce qui correspond avec le «bahir-anga-paribhāṣā » dont l'interprétation a présenté beaucoup de problèmes depuis le XIX ${ }^{\mathrm{e}}$ siècle (voir les discussions de ce paribhāṣā par Kielhorn et, un siècle plus tard, par Bronkhorst).

\section{Narratif et rituel dans la prose védique : la Maitrāyan̄isaminhitā}

Une des grandes Samihitā du Yajur-Veda Noir (Kṛș̣̣a-Yajur-Veda), la MaitrāyaṇīSamihitā n'a pas profité des outils disponibles pour d'autres Samihitā : il n'y pas de 
commentaire de Sāyaṇa ou d'autres auteurs, il n'y a pas de Prātiśākhya, et il n'y a que deux Śrauta-sūtra (Mānava et Vārāha). À titre de comparaison, la Taittirīya-Saminhitā, la Samihitā la plus tardive du YV Noir, a été commentée par plusieurs auteurs, connaît un Prātiśākhya, et plusieurs écoles rituelles (avec leurs Śrauta-sūtra, etc.) lui sont associées : celle de Baudhāyana, d'Āpastamba, de Bhāradvaja, d'Hiraṇyakeśi-Satyāṣậ̣ha. Parmi eux, Âpastamba est aujourd'hui le plus répandu. Comme nous l'avons vu l'année dernière, du point de vue de la langue, la Maitrāyaṇi-Samihitā se distingue surtout par des sandhis particuliers et par un vocabulaire parfois exceptionnel. La Maitrāyaṇ̄Samhitā est aussi importante pour ses mythes qui sont parfois sans aucun parallèle dans les texts voisins, et pour les formes du rituel védique qu'elle présuppose. Comme chaque Samihitā du Yajur-Veda, la Maitrāyaṇi-Samihitā est un Veda de l'Adhvaryu, le prêtre-gérant du sacrifice (tandis que le Hotar récite, l'Udgātar chante, le Brahmán observe, donne sa permission pour commencer les actes rituels et, si nécessaire, corrige les erreurs rituels).

Cette année nous étudiions une section de la Maitrāyaṇi-Samhitā, appelée gonāmikah, qui ouvre une perspective importante sur l'histoire ancienne du peuple védique. Depuis le $\mathrm{XIX}^{\mathrm{e}}$ siècle, les indianistes ont trouvé en Inde une grande littérature - la littérature védique - sans localisation géographique ni chronologie certaines, ainsi que des grandes cultures archéologiques - la céramique grise (Painted Grey Ware), par exemple - sans inscriptions ni textes. La littérature védique présuppose un rituel védique, à partir d'environ 1800-1500 av. notre ère (rituel Rggédique ancien), qui continue jusqu'à nos jours avec des écoles védiques installées depuis plusieurs siècles surtout dans le Sud de l'Inde. En dépit des efforts de plusieurs générations de spécialistes, on n'a pas réussi à mettre la main sur les « origines » de la littérature et de la culture védiques en Inde, ni sur la structure sous-jacente de ses liens visibles avec plusieurs littératures et cultures asiatiques et européennes. Pourtant, même si les résultats des recherches de ces spécialistes ne donnent aucun scénario concret de façon positive, ils permettent d'exclure un certain nombre d'hypothèses et de restreindre l'éventail des scénarios possibles. Par cette voie d'exclusion il est possible de suivre de loin, sur la base d'indications qui dérivent de plusieurs domaines de recherche, une présence surtout non-sédentaire des peuples indo-européens en Asie centrale, et, après $1800 \mathrm{av}$. notre ère, des tribus védiques dans le Nord-Ouest du sous-continent indien ayant établi un rituel védique qui nous est accessible par les sources textuelles. Ces tribus védiques se mettent en route vers la région centrale et le Nord-Est de l'Inde - jusqu'au $\mathrm{vI}^{\mathrm{e}}$ s. av. notre ère, lorsque le paysage s'est profondément transformé : c'est le début de l'urbanisation de la grande plaine du Gange, ce qui coïncide avec le début des religions urbaines du jainisme et du bouddhisme. Ces deux religions « protestantes » s'opposent à plusieurs aspects de la religion védique, parmi lesquels la célébration de l'abondance rurale dans de vastes sacrifices de chèvres, de bovins et de chevaux. Crucial, dans cette nouvelle conceptualisation de la période védique et ses antécédents - conceptualisation à laquelle ont contribué des spécialistes comme M. Witzel, H. Falk, S. Ratnagar et J. Bronkhorst -, c'est le mode de vie qu'on attribue aux tribus védiques : ils n'étaient ni des pastoralistes, dont la subsistance est basée principalement sur les produits de leurs troupeaux de vaches, chèvres, etc.; ni des agriculteurs qui se nourrissent des produits de la terre cultivée; ni des agriculteurs mixtes qui combinent, selon les cir- 
constances, la culture de céréales, etc. et l'élevage de bétail. Par contre, leur mode de vie représente ce qu'on peut appeler l'agro-pastoralisme, dont les traits caractéristiques sont (suivant un exposé récent de Ratnagar 2006) : 1) ceux qui pratiquent l'agropastoralisme apprécient les animaux domestiques beaucoup plus que les produits de l'agriculture, même s'ils consomment plus de produits céréaliers que de produits de l'élevage (lait, viande, etc.); 2) ils investissent les résultats positifs de leurs récoltes dans la croissance de leurs troupeaux; ces deux traits sont à la base d'un phénomène lié : 3) leur tendance à l'expansion géographique. Des indications pour 1) et 3) sont abondantes dans les anciens textes védiques et, volontairement négligés, les rituels védiques. Ce mode de vie du peuple védique une fois postulé, on pourrait expliquer plusieurs points énigmatiques des recherches menées jusqu'ici : leurs traces archéologiques sont minimales; ni les squelettes trouvés ni la composition génétique de la population indiquent une invasion significative; il y avait, par contre, une expansion graduelle sans que des batailles spectaculaires ont laissé des traces, même pas dans les textes (on ne compte pas ici les escarmouches intestines auxquelles plusieurs textes védiques et épiques renvoient); cette expansion graduelle se faisait dans tous les sens mais surtout vers l'est, où il y avait des vastes régions propices à la cultivation agropastoraliste, des régions forestières éparsement peuplées par des chasseurs-cueilleurs. La section de la Maitrāyaṇī-Samhitā que nous étudiions nous apporte une perspective unique - il n'y a pas de parallèles de cette section dans d'autres Samhitās - sur la vie dans un milieu agro-pastoraliste qui est encore loin de la confrontation avec l'urbanisme. La section présente l'image d'une vache « cosmique » :

de cette (vache), la terre est un pied; l'espace intermédiaire est un pied; le ciel est un pied; l'année est un pied; mais on dit aussi : l'agriculture est un pied (à elle, la vache cosmique); parfois elle se repose sur lui, parfois pas; quand il y a une bonne récolte, alors elle se repose (sur ce pied); quand il n'y a pas de récolte, elle ne se repose pas (sur ce pied; elle n'est pas bien établie, est instable).

Les rites indiqués, sur lesquelles nous avons des informations supplémentaires dans d'autres textes, par exemple, le Mānava-śrautasūtra, ne se comprennent que dans un contexte fondamentalement agro-pastoraliste. Les passages pertinents ont été également présentés et étudiés.

\section{Ayurveda : textes pré-modernes}

Parmi les systèmes de savoir transmis dans des traditions vivantes sur le souscontinent indien, l'Āyurveda, la « science de longévité » de l'Inde, occupe une place prééminente. Sa langue préférée était la variété de haut statut de l'indo-aryen qui s'appelait encore $b h \bar{a} s \bar{a}$ au deuxième siècle avant notre ère (dans l'œuvre des grammairiens tels que Pāṇini et Patañjali), et obtenait l'appellation samiskrta «polie, raffinée (à savoir, vacas, la parole) », c.-à-d., le " sanskrit », au cours des premiers siècles de notre ère. La cohésion et la vitalité de l'Āyurveda traditionnel (et de plusieurs autres systèmes de savoir indiens) pendant plus de deux millénaires sont attestées par la transmission soigneuse d'un nombre limité de textes classiques, un canon, parallèlement à la production créatrice de nouveaux textes qui reconnaissent et confir- 
ment - qui confirment parfois justement par la mise en question - le statut classique du canon. Les textes classiques disponibles présupposent une littérature antérieure en grande partie perdue. Les textes postérieurs ajoutent des détails et des modifications à un fond légendaire d'anciens textes. Les trois textes classiques principaux, dont le plus tardif, l'Așțānga-hrodaya de Vāgbhaṭa, a été daté du VII ${ }^{\mathrm{e}}$ siècle de notre ère, ont été préalablement édités, mais jusqu'ici pas de façon suffisamment critique; les traductions et les études sont donc également seulement préliminaires. En outre, les phases postérieurs des systèmes de savoir exigent une étude approfondie qui n'est pas moins urgente : premièrement, parce que le canon classique est transmis et même façonné par des processus ayant lieu au cours de ces périodes ultérieures; deuxièmement, parce que ces phases ultérieures relativement peu étudiées des systèmes de savoir préparent l'étape des transitions profondes dans la vie culturelle et intellectuelle de l'Inde, lorsqu'elle est rattrapée par les puissances coloniales, de la fin du XVII ${ }^{\mathrm{e}} \mathrm{s}$. jusqu'au XIX ${ }^{\mathrm{e}}$ et début du XX $\mathrm{XX}^{\mathrm{e}}$. Le texte choisi pour le cours, l'Abhinavacintāmaṇi de Cakrapāṇidāsa, a reçu peu d'attention des spécialistes, et les indications sur son étendue et sa date sont contradictoires. Au cours du séminaire, nous avons pu confirmer que la date correcte de son achèvement est, selon l'expression cryptique dans le dernier vers śālivāhanabhüpasya bhü-netra-muni-bhümite / abde kārttika-rākāyāim grantho'yaì pūrṇatāim gatah, l'automne (à la pleine lune de Kārttika) de 1721 dans l'ère de Śālivāhana, ce qui correspond à 1799 notre ère. Le texte est de caractère encyclopédique et consiste de soixante-six chapitres qui comprennent un total de 5342 vers. Le nombre beaucoup plus élevé de 10125 vers (Rahman et al., dans Science and Technology in Medieval India, 1982, p. 42) doit alors être rejeté; même le nombre de 5342 est trop élevé car les strophes de mètres longs comme le Sārdūlavikrīḍita ( 4 × 19 syllabes $)$ et la Sragdharā ( $4 \times 21$ syllabes) sont normalement coupées en deux par les éditeurs qui se sont intéressés pour le contenu médical plutôt que pour la forme poétique du texte. Dans le cours nous étudiions et traduisions une grande partie du premier chapitre qui sert d'introduction à l'œuvre et qui discute, parmi d'autres sujets, les caractéristiques d'un bon médecin et ceux d'un mauvais médecin, les unités de mesure et les règles générales sur les potions et pâtes. 